\title{
High prevalence and heterogeneity of Dysglycemia in patients with tuberculosis from Peru: a prospective cohort study
}

\author{
Roger I. Calderon ${ }^{1,2^{*}+}$ (D, Maria B. Arriaga ${ }^{3,4,5,6+}$, Kattya Lopez ${ }^{1}$, Nadia N. Barreda', Oswaldo M. Sanabria', \\ José F. Fróes Neto ${ }^{6,7}$, Davi Neri Araújo ${ }^{3,6}$, Leonid Lecca ${ }^{1,8}$ and Bruno B. Andrade $3,4,5,6,7,9,10^{*}$
}

\begin{abstract}
Background: The accuracy of different laboratory tests for diagnosis of diabetes mellitus (DM) and prediabetes (preDM) in populations exposed to tuberculosis (TB) remains poorly understood. Here, we examined the prevalence of DM and preDM in TB affected people in Lima, Peru.

Methods: A prospective cohort study of patients affected TB and their household contacts (HHC), was conducted between February and November 2017 in Lima, Peru. Fasting plasma glucose (FPG), HbA1c and oral glucose tolerance test (OGTT) were used to detect DM and preDM in a prospective cohort of TB patients $(n=136)$ and household contacts $(n=138)$. Diagnostic performance of the laboratory tests was analyzed. Potential effects of sociodemographic and clinical factors on detection of dysglycemia were analyzed.
\end{abstract}

Results: In TB patients, prevalence of DM and preDM was 13.97 and 30.88\% respectively. Lower prevalence of both DM (6.52\%) and preDM (28.99\%) were observed in contacts. FPG, HbA1C and OGTT had poor agreement in detection of preDM in either TB cases or contacts. TB-DM patients had substantially lower hemoglobin levels, which resulted in low accuracy of $\mathrm{HbA} 1 \mathrm{c}$-based diagnosis. Classic sociodemographic and clinical characteristics were not different between TB patients with or without dysglycemia.

Conclusion: High prevalence of DM and preDM was found in both TB patients and contacts in Lima. Anemia was strongly associated with TB-DM, which directly affected the diagnostic performance of HbA1c in such population.

Keywords: Diabetes mellitus, Prediabetes, Tuberculosis, Comorbidity, Prevalence

\section{Background}

Tuberculosis kills $1-2$ million people per year, especially in low and middle-income countries, and despite recent efforts to improve control programs, its incidence is declining at slow rates [1]. High-risk groups undermine the success of TB programs in the reducing the disease burden notwithstanding targeted interventions. One relevant aspect is diabetes mellitus (DM), which increases the risk of developing active TB in approximately three times [2]. More recently, prediabetes (preDM) has been also described as a risk factor for developing TB [3, 4]. Both the

\footnotetext{
*Correspondence: rcalderon_ses@pih.org; bruno.andrade@fiocruz.br ${ }^{\dagger}$ Roger I. Calderon and Maria B. Arriaga contributed equally to this work. ${ }^{1}$ Socios En Salud Sucursal Peru, 15001 Lima, Peru

${ }^{3}$ Faculdade de Medicina, Universidade Federal da Bahia, Salvador, Bahia 40110-100, Brazil

Full list of author information is available at the end of the article
}

World Health Organization (WHO) as the Peruvian National TB Program (NTP) recommend screening for DM in people with active TB and for TB between household contacts [5]. Despite those indications, most individuals are unaware of their DM or pre-DM status. In 2017, the Peruvian NTP communicated a DM incidence of $6.2 \%$ with a testing coverage of $77.9 \%$ of all TB patients whereas other instances of the national government reported in 2016 a DM incidence around 10.4\% (government communication). Those differences may reflect several limitations in the DM screening such as the use of only fasting plasma glucose (FPG) as the screening approach [5]. It is widely known that sensitivity of DM tests (such as HbA1c, fasting glucose and oral glucose tolerance) is variable [6-9]. It has been reported that $\mathrm{HbA1c}$ detects more people with DM [10] (or preDM) with higher sensitivity than other

(c) The Author(s). 2019 Open Access This article is distributed under the terms of the Creative Commons Attribution 4.0 International License (http://creativecommons.org/licenses/by/4.0/), which permits unrestricted use, distribution, and reproduction in any medium, provided you give appropriate credit to the original author(s) and the source, provide a link to the Creative Commons license, and indicate if changes were made. The Creative Commons Public Domain Dedication waiver (http://creativecommons.org/publicdomain/zero/1.0/) applies to the data made available in this article, unless otherwise stated. 
screening tests [11], so systematic use of FPG as screening tool, as observed in Peru, may result in incomplete record of TB-DM cases [12].

Due the discrepancies in sensitivity of DM screening tests and in order to determine the real prevalence of $\mathrm{DM}$ or preDM in TB patients and their household contact $(\mathrm{HHC})$, we conducted a study in Lima, Peru, with the hypothesis that both DM and pre-DM are more frequent than previously officially reported. In addition, we compared the performance of different screening methods to detect DM and preDM in TB cases. Furthermore, we examined the associations between different other clinical and sociodemographic risk factors with the possible more extended comorbid TB-DM burden.

\section{Methods}

\section{Study design}

This was a prospective cohort study of patients affected TB and their household contacts (HHC), conducted between February and November 2017 in Lima, Peru.
The recruitment details, as well as the procedures and investigations performed are outlined in Fig. 1a-b.

\section{Study population}

The study was carried out in the Public Hospital Sergio Bernales and outpatient health centers of Carabayllo and Comas districts. Patients with pulmonary TB with $\geq 16$ years of age diagnosed by the NTP of public health centers, who are not receiving anti-TB treatment or have started it in a period of no more than 5 days, were included. In this study, HHC was defined as a person 12 years of age or older who shared at least household where they sleep or take their meals (at least one of them per day) with a study TB index patient. Exclusion criteria were patients or contacts diagnosed with HIV, pregnant women, who did not live permanently in the jurisdiction area of the study and patients who had infection or disease due to non-tuberculous mycobacteria. The follow-up of those patients was conducted up to 6 to 12 months after enrollment.

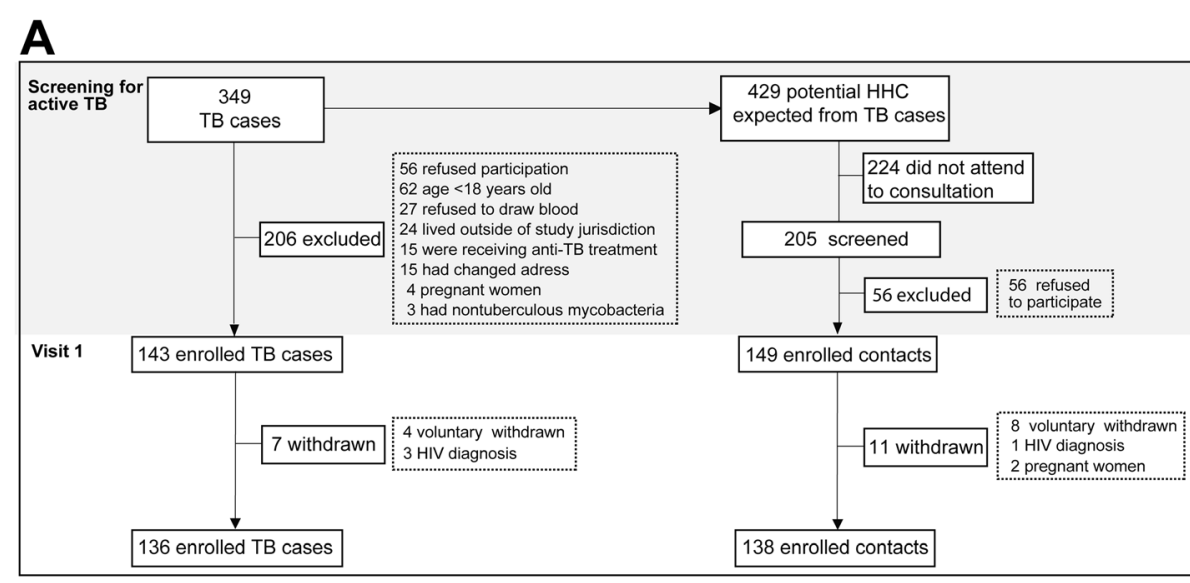

B

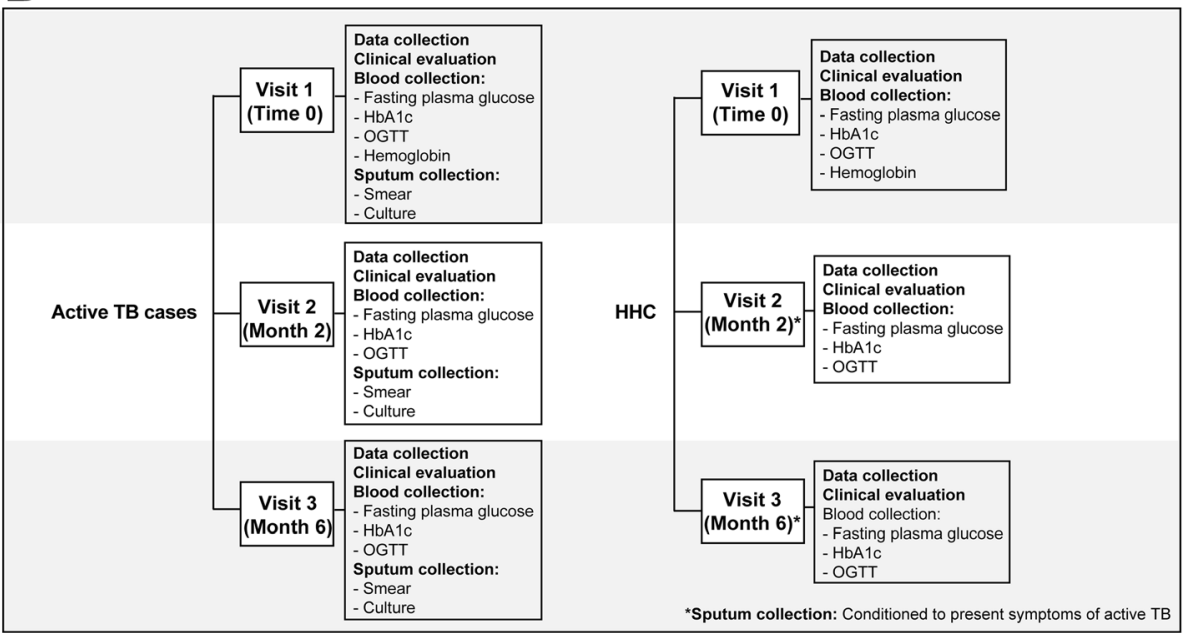

Fig. 1 Study outline. a Flowchart describing the recruitment of the study population. b Flowchart of the investigations and procedures performed 


\section{Study power and sample size calculations}

Power calculations were performed using the software OpenEpi 3.0 (www.openepi.com). We expected that 130 individuals per group (TB cases and $\mathrm{HHC}$ ) would result in about $80 \%$ of power to detect difference in DM prevalence of $10 \%$ ( $14 \%$ in TB cases and $4 \%$ in $\mathrm{HHC}$ ), at a significance level of $\alpha=0.05$.

\section{Laboratory and field procedures}

One sputum specimen for each patient was isolated for smear investigation (acid-fast bacilli, AFB) and well as Mycobacterium tuberculosis (Mtb) solid cultures. For AFB investigation, sputum smears were stained with ZiehlNeelsen and examined by microscopy. The remaining sputum samples were cultured for Mtb at baseline and different study visits until completion of anti-TB treatment. Positive smears of Mtb were graded as 1+ (10-99 AFB observed in 100 fields), $2+$ ( 1 a 10 AFB in 50 fields) and $3+$ (more than 10 AFB in 20 fields). For observations up to 9 AFB, exact number of AFB was recorded as previously described [13]. Mtb positive cultures were graded as $1+$ (20-100 colonies), 2+ (more than 100 discrete colonies) and 3+ (more than 100 colonies forming a confluent mass). For growth of up to 19 colonies, exact number of colonies was recorded as previously described [14]. Mtb complex and drug susceptibility testing were performed following standard procedures reported elsewhere [15]. All the TB testing were performed at the Socios En Salud (SES) Laboratory located in Lima.

DM was defined in agreement with American Diabetes Association (ADA) guidelines [16] as 2-h glucose $\geq 200$ $\mathrm{mg} / \mathrm{dL}$ (OGTT), HbA1c $\geq 6.5 \%$ or fasting plasma glucose $\geq 126 \mathrm{mg} / \mathrm{dL}$. PreDM was also defined in agreement with ADA guidelines as 2-h glucose 140 a $199 \mathrm{mg} / \mathrm{dL}, \mathrm{HbA} 1 \mathrm{c}$ 5.7 a $6.4 \%$ or fasting plasma glucose $100-125 \mathrm{mg} / \mathrm{dL}$. The measurement of HbA1c in whole blood specimens was performed by use of TRI-stat ${ }^{\text {tw }}$ platform (Trinity Biotech, Ireland) and fast serum glucose or glucose level on OGTT test, was performed following standard methods. Of note, OGTT was performed only in individuals without prior DM diagnosis following instructions from the Institutional Review Board which handled the Ethical aspects of the study protocol.

Anemia was defined following WHO criteria as hemoglobin $(\mathrm{Hb})$ level below $12.5 \mathrm{~g} / \mathrm{dL}$ for female and $13.5 \mathrm{~g} / \mathrm{dL}$ for male. $\mathrm{Hb}$ measurement was performed in whole blood specimens stored at $-80^{\circ} \mathrm{C}$ from patients and $\mathrm{HHC}$ around one year after the blood sample collection at the end of the enrollment. One $50 \mu \mathrm{L}$-aliquot of whole blood of each participant was thawed and separated in a new tube to be passed through the HumaCount 5D Hematology System (Wiesbaden, Germany).

The SES lab conducts annual external quality assurance through competition panels of the College of
American Pathologists (Northfield, Illinois) and other agencies.

\section{Clinical data}

Clinical evaluations were also carried out by a specialist, interviews for the collection of socio-demographic and clinical information, review of medical records to obtain relevant clinical information on comorbidities such as blood pressure, pulse, respiratory rate, immunosuppressive conditions, among others. Hypertension defined by WHO criteria: measured on two different days, the systolic blood pressure readings on both days is $\geq 140$ $\mathrm{mmHg}$ and/or the diastolic blood pressure readings on both days is $\geq 90 \mathrm{mmHg}$. The anthropometric measurement was performed: Weight, height and abdominal circumference measurement. Demographic and clinical information were registered in the Socios En Salud Informatic System (SEIS) software (Lima, Peru).

\section{Outcomes}

Treatment outcome was classified as successful for patients considered microbiologically "cured" or (negative Mtb cultures and AFB negative in sputum smears at the end of treatment), whereas "death", "default" (treatment dropout/abandonment) and "failure" (positive cultures at the end of treatment) were combined as poor outcome [17]. The primary outcome in this study was the prevalence of $\mathrm{DM}$ and preDM in patients with $\mathrm{TB}$ and their HHC using the fasting serum glucose assays, HbA1c or OGTT (this one only in people without prior DM diagnosis), evaluated and indicated by the endocrinologist and also following the considerations of the American Diabetes Association.

\section{Data analysis}

We assessed the diagnostic performance of FPG and $\mathrm{HbA1c}$ for DM and preDM using the conjunction of OGTT testing and clinical DM/preDM diagnosis, both as gold standard. Characteristics of study participants were presented as median and interquartile ranges (IQR) for continuous variables or frequency for categorical variables. Continuous variables were compared using the MannWhitney $U$ test (between two groups) or Kruskal-Wallis test with Dunn's multiple comparisons. Categorical variables were compared using the Fisher's exact test $(2 \times 2$ comparisons) or Pearson's chi square test. We constructed Receiver Operating Characteristic (ROC) curves to calculate sensitivity, specificity, and predictive values determined at different cut-off values for HbA1c and FPG. Finally, the Kappa (K) statistic was calculated to assess agreement between OGTT versus FPG or HbA1c as diagnostic test for DM or preDM. The Kappa statistic was interpreted by Landis and Koch criteria [18]. All analyses were prespecified. Two-sided $P$ value $<0.05$ after adjustment for 
multiple comparisons (Bonferroni's method) were considered statistically significant. Statistical analyses were performed using SPSS 24.0 (IBM statistics), Graphpad Prism 7.0 (GraphPad Software, San Diego, CA) and JMP 13.0 (SAS, Cary, NC, USA).

\section{Results}

We initially screened 349 microbiologically-confirmed TB cases at the primary health care centers which were part of the present study, between February and November 2017. During the screening, 206 individuals were excluded for a number of reasons listed in Fig. 1a, and 143 patients with active TB were examined in the first study visit (Fig. 1a and Fig. 1b). At this stage, additional 7 persons were excluded due to HIV diagnosis $(n=3)$ and consent withdraw $(n=4)$, resulting in a cohort of 136 patients. During the search of HHCs of the TB index cases, 205 people were identified for screening and only 149 were effectively screened. Of those, 8 withdrew consent and 3 were excluded for having positive HIV status $(n=1)$ or pregnancy $(n=2)$ resulting in a total of $138 \mathrm{HHC}$ participants (Fig. 1).
At enrollment, the DM prevalence was $13.97 \%$ (95\% CI: $8.14,19.80 \%)(n=19)$ among TB patients (all of whom refereed DM diagnosed prior to study enrollment), while the prevalence of DM among $\mathrm{HHC}$ was 6.52\% (95\% CI: 2.40, $10.64 \%)(n=9)$. The prevalence of preDM was $30.88 \%(95 \%$ CI: $23.81,38.65 \%)$ in TB patients $(n=42)$ and $28.99 \%(95 \%$ CI: 21.42, 36.56\%) among the HHC $(n=40)$. Diagnosis of preDM was performed at study enrollment. Comparisons at this timepoint revealed that TB and HHC groups exhibited similar frequencies of DM and preDM (Fig. 2a). After 2 months of antitubercular treatment (ATT) commencement, DM frequency was nearly double in TB vs. HHC whereas preDM was higher in the latter group (Fig. 2a). At month 6 of ATT, frequency of DM and preDM was once again not different between the study groups, although there was a remarkable $75 \%$ loss to follow-up in the HHC group at this timepoint (Fig. 2a).

Among the TB index cases at study baseline, DM individuals were on average older than preDM and normoglycemic individuals (median age: $46.41 \mathrm{yrs}$. IQR:33.554.8 vs. 39.8 yrs., IQR: $26.7-54.0$ and 26.4 yrs. IQR: 22.3-34.7, respectively, $p<0.01$ ) (Table 1 ). In addition,

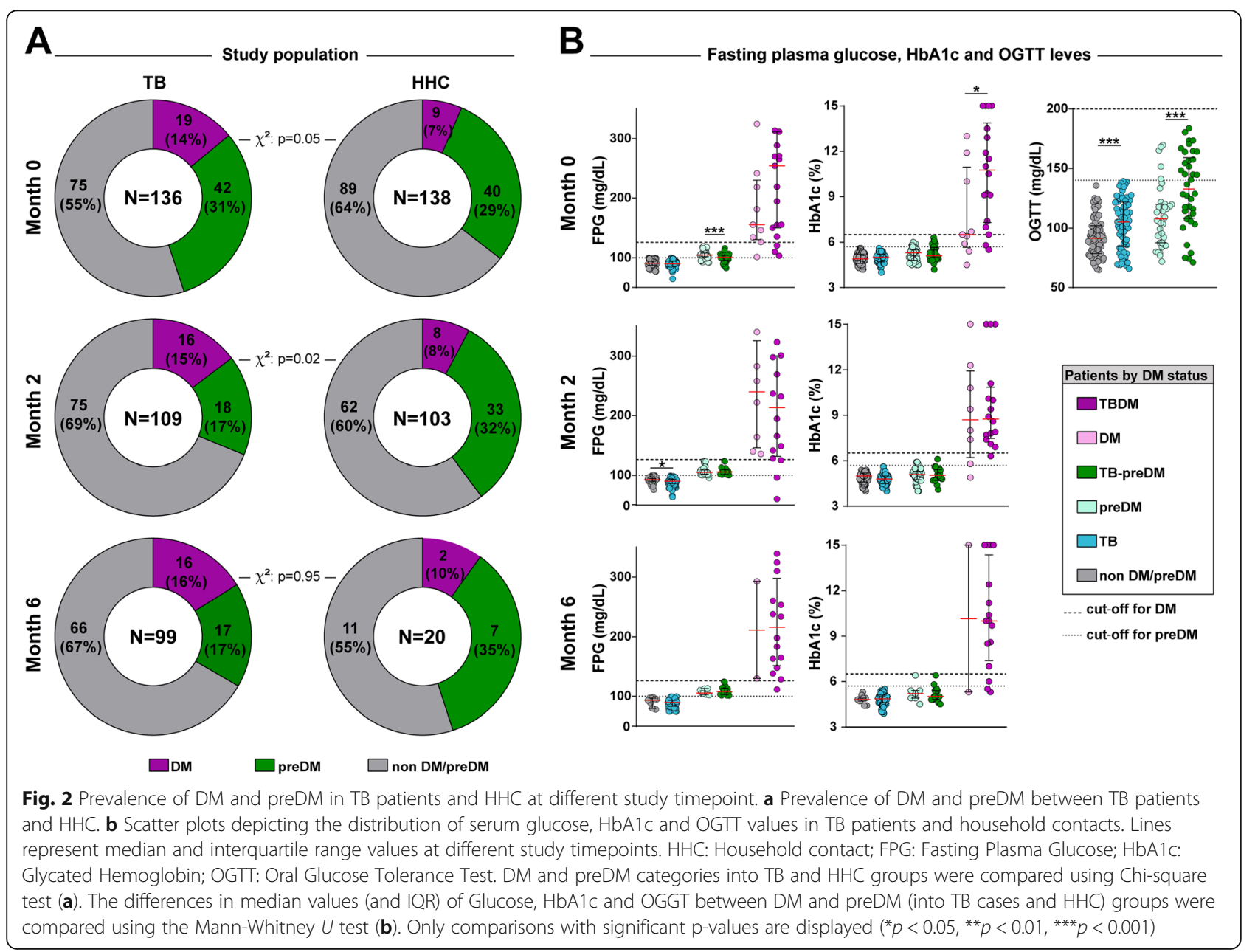


Table 1 Characteristics of pulmonary TB cases stratified according to glycemic status

\begin{tabular}{|c|c|c|c|c|c|}
\hline \multirow[t]{2}{*}{ Characteristics } & \multirow[t]{2}{*}{$\mathrm{n} / \mathrm{N}$} & \multirow{2}{*}{$\begin{array}{l}\text { TBDM } \\
n=19\end{array}$} & \multirow{2}{*}{$\begin{array}{l}\text { TBpreDM } \\
n=42\end{array}$} & \multirow{2}{*}{$\begin{array}{l}\text { Normoglycemic } \\
n=75\end{array}$} & \multirow[t]{2}{*}{$p$-value } \\
\hline & & & & & \\
\hline Age (years)-median (IQR) & 136/136 & $46.41(33.5-54.8)$ & $39.8(26.7-54.0)$ & $26.4(22.3-34.7)$ & $<0.01$ \\
\hline Gender & 136/136 & & & & 0.354 \\
\hline Male & $136 / 136$ & $9(47.4)$ & $27(64.3)$ & $47(62.7)$ & \\
\hline Female & $136 / 136$ & $10(52.6)$ & $15(35.7)$ & $28(37.3)$ & \\
\hline Prior TB & $136 / 136$ & $5(26.3)$ & $8(19.0)$ & $10(13.3)$ & 0.158 \\
\hline BCG vaccination & $135 / 136$ & $17(89.5)$ & $38(92.7)$ & $71(94.7)$ & 0.411 \\
\hline Smoking & $135 / 136$ & $4(21.1)$ & $10(24.4)$ & $15(20.0)$ & 0.767 \\
\hline Smokers at home & $135 / 136$ & $2(10.5)$ & $4(9.8)$ & $5(6.7)$ & 0.499 \\
\hline Cannabis use & $135 / 136$ & $1(5.3)$ & $7(17.1)$ & $13(17.3)$ & 0.283 \\
\hline Illicit drug use & $135 / 136$ & $2(10.5)$ & $7(17.1)$ & $8(10.7)$ & 0.707 \\
\hline Alcohol use & $135 / 136$ & $4(21.1)$ & $29(70.7)$ & $37(49.3)$ & 0.348 \\
\hline Hypertension & $135 / 136$ & $4(21.1)$ & $3(7.3)$ & $0(0.0)$ & $<0.01$ \\
\hline Asthma & $135 / 136$ & $0(0.0)$ & $3(7.3)$ & $5(6.7)$ & 0.399 \\
\hline Renal disease & 135/136 & $1(5.3)$ & $0(0.0)$ & $2(2.7)$ & 0.844 \\
\hline Slow scarring & $135 / 136$ & $3(15.8)$ & $9(22.0)$ & $7(9.3)$ & 0.186 \\
\hline Metformin use & 132/136 & $7(36.8)$ & $0(0.0)$ & $0(0.0)$ & $<0.01$ \\
\hline BMI (kg/m2)-median (IQR) & 132/136 & $22.3(21.4-26.4)$ & $23.2(21.2-25.1)$ & $22.3(20.3-25.4)$ & 0.749 \\
\hline Waist circumference (cm) -median (IQR) & 132/136 & $85(81-89)$ & $88(80-91)$ & $83(76-88)$ & 0.058 \\
\hline $\mathrm{Hb}(\mathrm{g} / \mathrm{dL})$-median (IQR) & $134 / 136$ & $9.9(7.6-13.4)$ & $12.0(10.8-13.1)$ & $12.6(11.3-13.4)$ & \\
\hline Anemia & $136 / 136$ & $19(100)$ & $31(73.8)$ & $49(66.2)$ & 0.006 \\
\hline FPG (mg /dL) -median (IQR) & $136 / 136$ & $218.9(147.7-298.1)$ & $103.1(100.4-106.3)$ & $89.7(85.6-93.9)$ & $<0.01$ \\
\hline HbA1c (\%)-median (IQR) & $134 / 136$ & $10.8(7.4-13.5)$ & $5.1(4.8-5.5)$ & $5.00(4.6-5.1)$ & $<0.01$ \\
\hline OGTT (mg/dL) -median (IQR) & 112/136 & $119.5(119.5-119.5)$ & $128.5(106.5-157.1)$ & $105.2(85.8-121.4)$ & $<0.01$ \\
\hline AFB smear & 135/136 & & & & 0.004 \\
\hline Negative & & $5(26.3)$ & $15(35.7)$ & $40(54.1)$ & \\
\hline $1+$ & & $3(15.8)$ & $9(21.4)$ & $15(20.3)$ & \\
\hline $2+$ & & $3(15.8)$ & $6(14.3)$ & $6(8.1)$ & \\
\hline $3+$ & & $7(36.8)$ & $10(23.8)$ & $11(14.9)$ & \\
\hline Scanty & & $1(5.3)$ & $2(4.8)$ & $2(2.7)$ & \\
\hline L-J culture & 131/136 & & & & 0.59 \\
\hline Negative & & $4(21.1)$ & $9(22.5)$ & $25(34.2)$ & \\
\hline $1+$ & & $12(63.2)$ & $18(45.0)$ & $33(45.2)$ & \\
\hline $2+$ & & $2(10.5)$ & $4(10.0)$ & $3(4.2)$ & \\
\hline $3+$ & & $0(0.0)$ & $4(10.0)$ & $3(4.2)$ & \\
\hline colonies & & $1(5.3)$ & $5(12.5)$ & $8(11.1)$ & \\
\hline BD MGITTM 960 System & $78 / 136$ & & & & 0.11 \\
\hline Positive & & $8(80.0)$ & $22(81.5)$ & $34(82.9)$ & \\
\hline Negative & & $2(20.7)$ & $5(18.5)$ & $7(17.1)$ & \\
\hline MDR & $76 / 136$ & $2(18.2)$ & $3(12.5)$ & $4(9.8)$ & 0.451 \\
\hline Isoniazid-resistant & $76 / 136$ & $2(18.2)$ & $5(20.8)$ & $7(17.1)$ & 0.831 \\
\hline Rifampicin-resistant & $76 / 136$ & $2(18.2)$ & $4(16.7)$ & $5(12.2)$ & 0.55 \\
\hline TB treatment outcome & $115 / 136$ & & & & 0.64 \\
\hline Poor & & $5(27.8)$ & $8(23.5)$ & $14(22.2)$ & \\
\hline
\end{tabular}


Table 1 Characteristics of pulmonary TB cases stratified according to glycemic status (Continued)

\begin{tabular}{|c|c|c|c|c|c|}
\hline \multirow[t]{2}{*}{ Characteristics } & \multirow[t]{2}{*}{$\mathrm{n} / \mathrm{N}$} & TBDM & \multirow{2}{*}{$\begin{array}{l}\text { TBpreDM } \\
n=42\end{array}$} & Normoglycemic & \multirow[t]{2}{*}{$p$-value } \\
\hline & & $n=19$ & & $n=75$ & \\
\hline Cure & & $13(72.2)$ & $26(76.5)$ & $49(77.8)$ & \\
\hline Polyuria & $136 / 136$ & $9(47.4)$ & $16(38.1)$ & $28(37.3)$ & 0.493 \\
\hline Polydipsia & $136 / 136$ & $9(47.4)$ & $20(47.6)$ & $36(48.0)$ & 0.956 \\
\hline
\end{tabular}

Data represent no. (\%); IQR: Interquartile range. BCG Bacillus Calmette-Guérin, BMI Body Mass Index, Hb Hemoglobin, FPG Fasting Plasma Glucose, HbA1c Glycated Hemoglobin, OGTT Oral Glucose Tolerance Test, AFB Acid-Fast Bacilli, L-J Löwenstein-Jensen, MDR Multi Drug Resistant. Hypertension, asthma, renal disease and anemia as defined by the World Health Organization as described in Methods. Prior TB: diagnosis of active tuberculosis before of this

lower level of education (primary and secondary school years) was associated with presence of dysglycemia (DM or preDM) $(p<0.01$; Table 1$)$. Hypertension was more frequently in DM patients (21.1\%) than that in normoglycemic TB cases $(7.3 \%)(p<0.01)$. The study groups were similar with regard to a number of other characteristics including sex, history of prior TB, asthma, renal disease and lifestyle habits (alcohol use, smoking and illicit drugs use) (Table 1).

When the cohort of $\mathrm{HHC}$ was stratified according to the glycemic status, we found once again that DM patients were on average older than those with preDM or normoglycemia (Table 2). In addition, the highest values of body mass index (BMI) and waist circumference were detected in the subgroup of preDM HHC (Table 2). Other characteristics were similar between the HHC subgroups.

The FPG and HbA1c tests were performed prospectively in all patients recruited (except OGTT for known DM cases). At the baseline visit, as expected, TB patients with coincident DM exhibited higher values of FPG and HbA1c than those diabetic but without TB (Fig. 2b). Such discrepancies in laboratory measurements were reduced at month 2 and 6 of ATT and no statistically significant differences were observed between diabetic patients with or without TB (Fig. 2b). The OGTT was performed only at baseline in patients without prior DM diagnosis. One individual was identified as having DM (from the HHC group). Once again, presence of TB was associated with higher values of OGTT results than in $\mathrm{HHC}$ individuals, suggesting that Mtb infection may drive inflammation-associated dysglycemia [19, 20] (Fig. 2b).

Moreover, the frequencies of TB-related symptoms between TB index cases with different degree of dysglycemia were compared and we found no statistically significant difference (Fig. 3a). Moreover, frequency patients presenting with increased acid-fast bacilli grades in sputum smears was higher in TB cases with DM compared to the other groups (chi-square $p=0.004$; Fig. 3b). These findings indicate that TB clinical presentation is not worse in diabetics as we have previously demonstrated in different studies from Brazil [3, 19], but confirmed previously reported data showing increased mycobacterial loads in sputum smears [19].

Circulating hemoglobin levels were low in diabetic patients with coincident TB compared to those with preDM or normoglycemia (Fig. 4a and b). In fact, within the group of TB, anemia was detected in all diabetics but only in $73.8 \%$ of preDM $(n=31)$ and $66.2 \%$ of normoglycemic patients $(n=49)(p=0.006)$. In $\mathrm{HHC}$, diabetes was not associated with substantial changes in hemoglobin levels (Fig. 4a and b). Moreover, frequency of anemia was not different between the groups of TB contacts presenting with diverse degree of dysglycemia $(p=0.08)$. This finding suggested that a potential synergistic effect of DM and $\mathrm{TB}$ on the degree of anemia may exist. Indeed, results from FPG and HbA1c in TB patients demonstrated that values observed in DM were substantially higher than in preDM or normoglycemia, and similar trend was observed in OGTT between preDM and nondiabetics (Fig. 4c). Of note, in $\mathrm{HHC}$, values of FPG and HbAlc were lower in anemic individuals compared to those with normal hemoglobin levels (Fig. 4c). Furthermore, OGTT levels were able to distinguish preDM from normoglycemia only in the subgroup of participants without anemia (Fig. 4c).

We next analyzed the overall accuracy of FPG and HbA1c in detecting DM or preDM cases in individuals stratified according to TB diagnosis (TB patients and HHC). FPG levels were able to detect more cases with preDM, but not with DM, than $\mathrm{HbA1c}$ did in the entire study population (Fig. 5a). When only TB cases were considered, again FPG was able to detect more preDM patients then HbA1c (Fig. 5b). Similar findings were observed in $\mathrm{HHC}$ (Fig. 5c). These results indicate that the laboratory tests examined here displayed different values of accuracy to detect DM or preDM in the study population. Summary of the accuracy and predictive values are shown in Additional file 1: Table S1. Concordance on DM diagnosis using either FPG or HbA1c levels was low, with lower performance of HbA1c (Fig. 6a). In addition, poor agreement was also observed between OGTT versus 
Table 2 Characteristics of household contacts of pulmonary TB cases stratified glycemic status

\begin{tabular}{|c|c|c|c|c|c|}
\hline \multirow[t]{2}{*}{ Characteristics } & \multirow[t]{2}{*}{$n / N$} & \multirow{2}{*}{$\begin{array}{l}\mathrm{DM} \\
n=9\end{array}$} & \multirow{2}{*}{$\begin{array}{l}\text { preDM } \\
n=40\end{array}$} & \multirow{2}{*}{$\begin{array}{l}\text { Normoglycemic } \\
n=89\end{array}$} & \multirow[t]{2}{*}{$p$-value } \\
\hline & & & & & \\
\hline Age (years) -median (IQR) & $138 / 138$ & $60.9(57.5-68.0)$ & $49.2(40.0-57.3)$ & $30.45(65.83)$ & $<0.01$ \\
\hline Gender & $136 / 138$ & & & & 0.44 \\
\hline Male & & $5(55.6)$ & $21(52.5)$ & $30(34.5)$ & \\
\hline Female & & $4(44.4)$ & $19(47.5)$ & $57(65.5)$ & \\
\hline Education & $138 / 138$ & & & & 0.207 \\
\hline Primary or secondary & & $9(100.0)$ & $33(82.5)$ & $71(79.8)$ & \\
\hline Technical or university & & $0(0.0)$ & $7(17.5)$ & $18(20.2)$ & \\
\hline Prior TB & $135 / 138$ & $2(22.2)$ & $3(7.7)$ & $9(10.3)$ & 0.618 \\
\hline BCG vaccination & $135 / 138$ & $9(100.0)$ & $36(92.3)$ & 84 (96.6) & 0.752 \\
\hline Smoking & $135 / 138$ & $0(0.0)$ & $7(17.9)$ & $12(13.8)$ & 0.682 \\
\hline Smokers at home & $135 / 138$ & $2(22.2)$ & $6(15.4)$ & $11(12.6)$ & 0.427 \\
\hline Cannabis use & $134 / 138$ & $0(0.0)$ & $2(5.1)$ & $0(0.0)$ & 0.185 \\
\hline Illicit drug use & $134 / 138$ & $0(0.0)$ & $1(2.6)$ & $0(0.0)$ & 0.35 \\
\hline Alcohol use & $134 / 138$ & $2(22.2)$ & $22(56.4)$ & $25(29.1)$ & 0.134 \\
\hline Hypertension & $135 / 138$ & $0(0.0)$ & $6(15.4)$ & $6(6.9)$ & 0.647 \\
\hline Asthma & $135 / 138$ & $0(0.0)$ & $3(7.7)$ & $7(8.0)$ & 0.515 \\
\hline Renal disease & $135 / 138$ & $0(0.0)$ & $3(7.7)$ & $4(4.6)$ & 0.978 \\
\hline Slow scarring & $135 / 138$ & $2(22.2)$ & $3(7.7)$ & $6(6.9)$ & 0.229 \\
\hline Metformin use & $133 / 138$ & $2(22.2)$ & $0(0.0)$ & $0(0.0)$ & $<0.01$ \\
\hline Consanguinity with index case & $135 / 138$ & $3(33.3)$ & $3(7.7)$ & $15(17.2)$ & 0.96 \\
\hline BMI (kg/m2) -median (IQR) & 133/138 & $29.41(26.9-31.7)$ & $29.8(28.1-33.4)$ & $26.1(2.9-29.4)$ & $<0.01$ \\
\hline Waist circumference $(\mathrm{cm})$-median (IQR) & $133 / 138$ & $95(93-105)$ & $98(94-107)$ & $88(79-94)$ & $<0.01$ \\
\hline $\mathrm{Hb}(\mathrm{g} / \mathrm{dL})$-median (IQR) & 138/138 & $13.2(12.9-13.7)$ & $13.6(12.8-14.6)$ & $13.1(12.1-14.2)$ & 0.151 \\
\hline Anemia & 138/138 & $2(22.2)$ & $8(20.0)$ & $35(39.3)$ & 0.138 \\
\hline FPG (mg /dL) -median (IQR) & $138 / 138$ & $155.60(134.3-218.3)$ & $104.6(102.0-107.9)$ & $90.9(87.5-94.2)$ & $<0.01$ \\
\hline HbA1c (\%)-median (IQR) & 138/138 & $6.5(5.9-10.0)$ & $5.3(4.8-5.50)$ & $4.9(4.6-5.2)$ & $<0.01$ \\
\hline OGTT (mg/dL) -median (IQR) & $124 / 138$ & $91.6(70.3-107.10)$ & $107.8(87.7-119.8)$ & $91.6(78.9-101.9)$ & $<0.01$ \\
\hline Polyuria & $138 / 138$ & $5(55.6)$ & $7(17.5)$ & $19(21.3)$ & 0.187 \\
\hline Polydipsia & $138 / 138$ & $3(33.3)$ & $14(35.0)$ & $19(21.3)$ & 0.124 \\
\hline Malaise & $138 / 138$ & $2(22.2)$ & $10(25.0)$ & $24(27.0)$ & 0.721 \\
\hline
\end{tabular}

Data represent no. (\%); IQR Interquartile range, BCG Bacillus Calmette-Guérin, BMI Body Mass Index, Hb Hemoglobin, FPG Fasting Plasma Glucose, HbA1c Glycated Hemoglobin, OGTT Oral Glucose Tolerance Test. Hypertension, asthma, renal disease and anemia as defined by the World Health Organization as described in Methods

HbA1c or FPG tests to detect preDM in both TB patients and HHC (Fig. 6b).

\section{Discussion}

Estimating the prevalence of dysglycemia among TB cases is important to understand the real burden of TBDM and drive changes to optimize detection and treatment of this comorbidity. In the present study, high prevalence of DM and preDM was found in both $\mathrm{TB}$ patients (14\%) and HHC (6.5\%), which is unexpectedly higher than previous reports from the Peruvian Minister of Health (6.2\%) [21], but similar to results from a recent report [22]. In the last years, low and middle income countries, such as Peru, have experienced a nutritional transition [23]. These populations historically affected by hunger and malnutrition now face additional problems of obesity and other non-communicable diseases, such as diabetes and hypertension [24]. Because DM is a known risk factor of $\mathrm{TB}[2,25,26]$, systematic screening should be performed in high-risk populations. Nevertheless, maybe due to high cost, many countries do not perform systematic screening and for this reason the dual burden of TB and DM is likely still underestimated. Findings from this study and others $[27,28]$ argue that, 

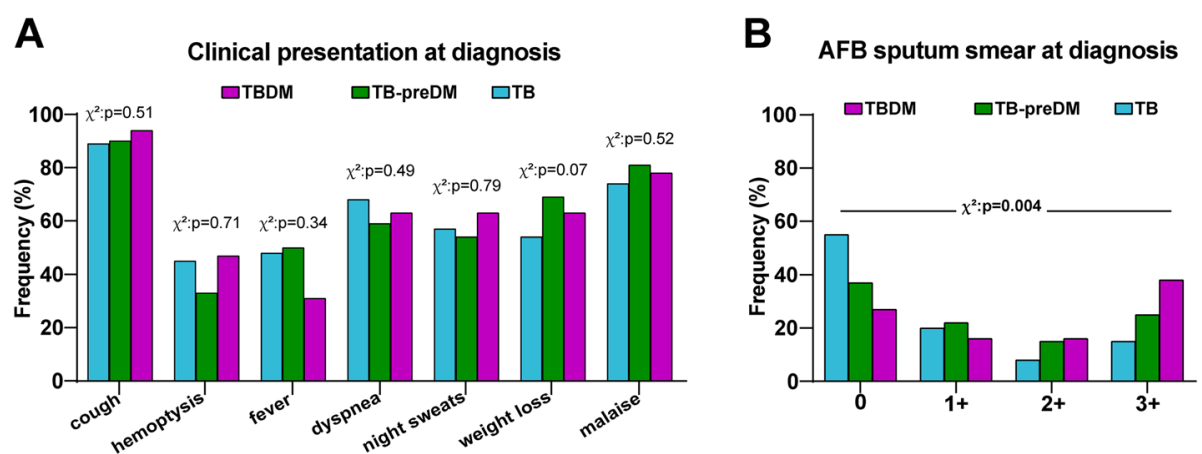

Fig. 3 TB-diabetes comorbidity is associated with increased mycobacterial loads in sputum smears, but in frequency of TB-related symptoms. a Prevalence of indicated TB-related symptoms was compared between TB patients with diabetes (TBDM), prediabetes or normoglycemia. b Frequency of TB patients with different AFB smear grades in sputum classified according to glycemic status as indicated. Data were analyzed using the Pearson's chi-square test

to provide a more reliable picture of the TBDM/preDM burden and based on some other references, it is necessary to consider the joint use of FPG and HbA1c in all patients, while OGTT could be used, when it is safe, to rule out prediabetes.

In our prospective evaluation, we found that frequency of DM remained stable during the follow up period, including in the group of TB patients. This could be due to the fact that most of the DM patients investigated exhibited high levels of HbA1c, compatible with uncontrolled diabetes [29] and these values remained high during the follow-up, in disagreement to results from a previous report [30]. We hypothesize that patients with very high levels of HbA1c are not able to substantially decrease values after ATT-induced reduction of systemic inflammation. On the other hand, frequency of preDM significantly reduced in $\mathrm{TB}$ patients undergoing ATT but were again stable in the follow up timepoint of the $\mathrm{HHC}$ cohort. This observation argues that resolution of systemic inflammation during ATT may lead to reduction of transient dysglycemia detected in some patients with TB.

There is evidence that TB clinical presentation is exacerbated in individuals $[25,31,32]$. In addition, poor glycemic control has been described to negatively impact radiographic manifestation of pulmonary disease in patients with TBDM

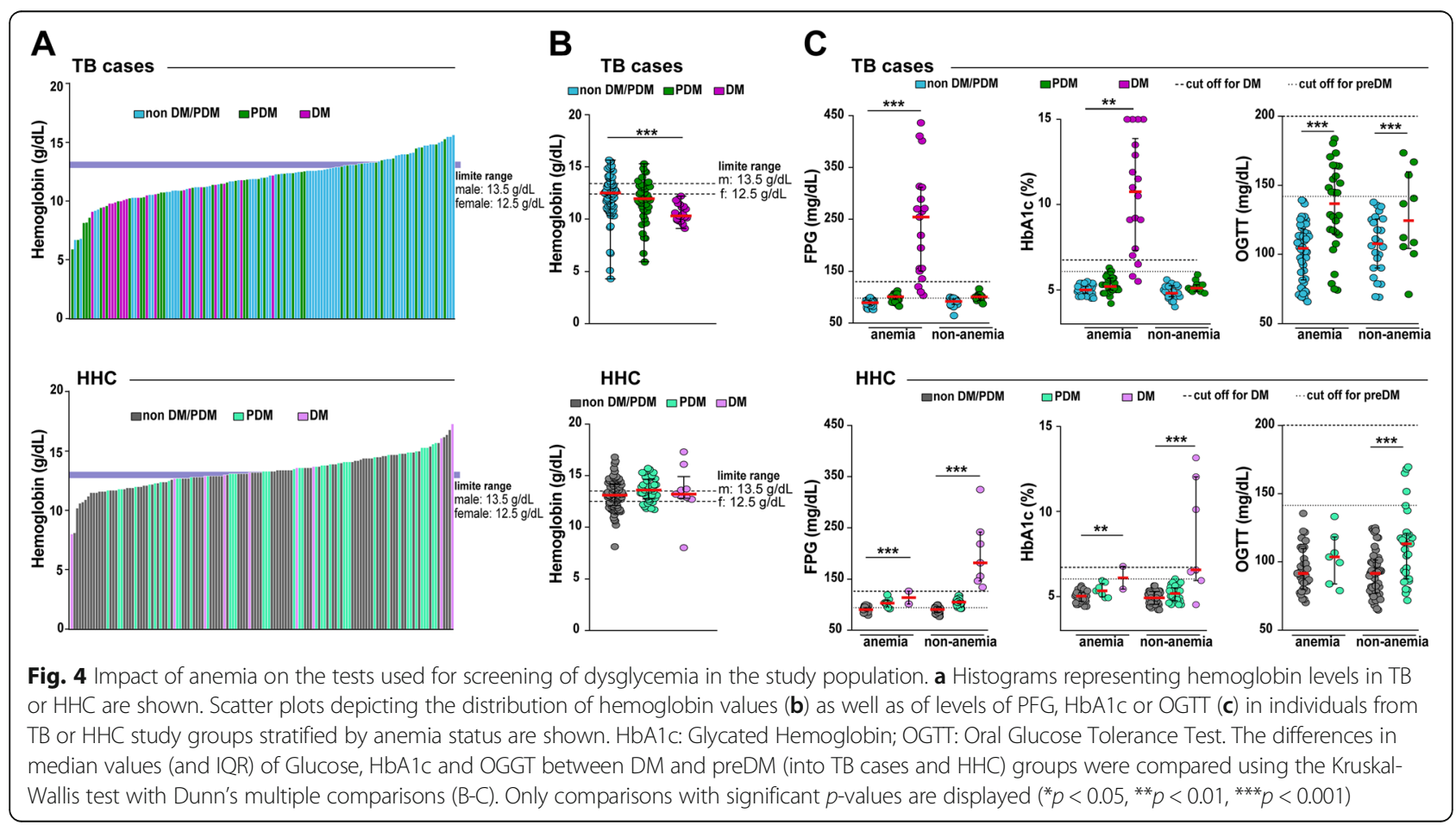




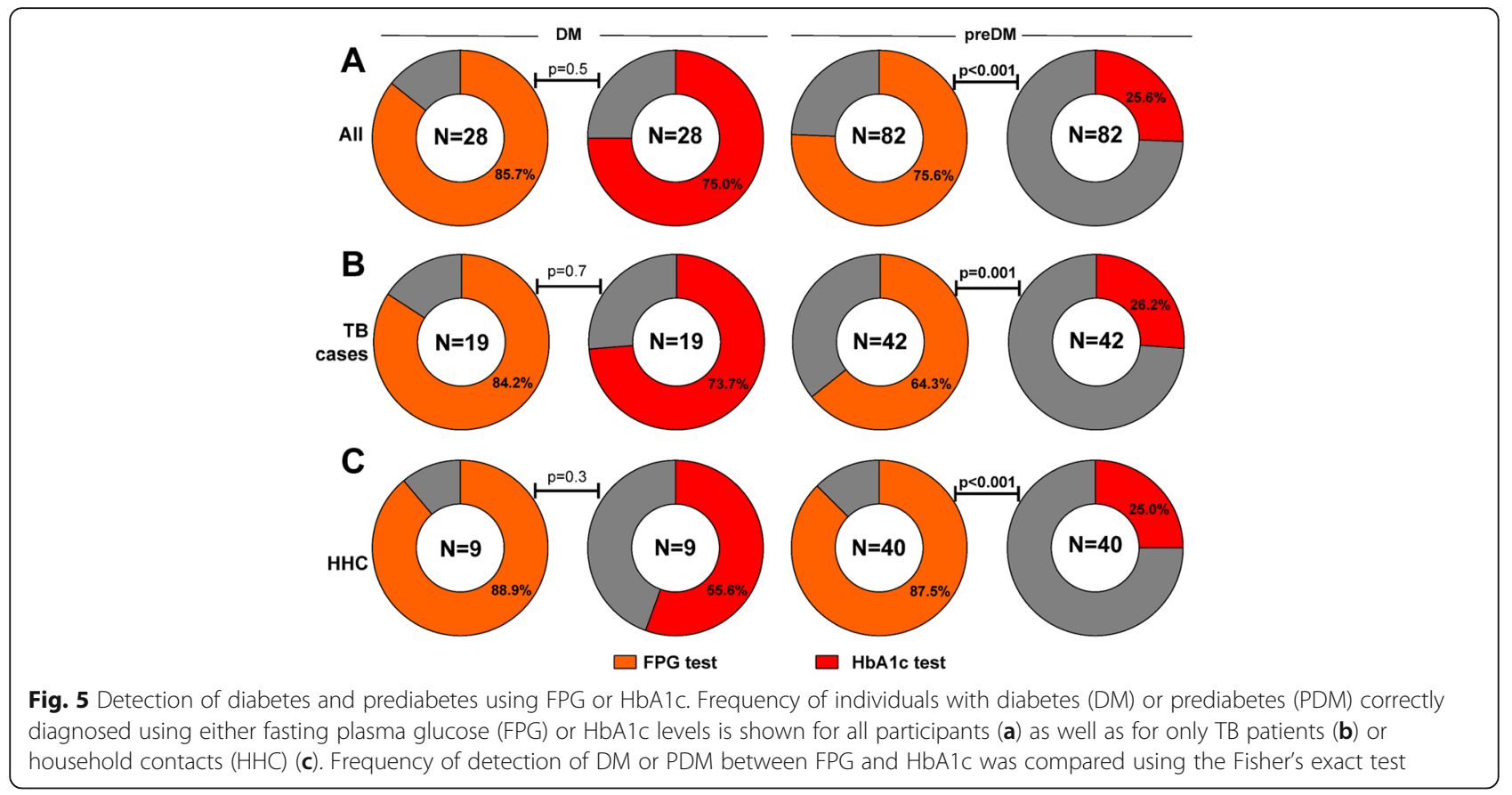

[25]. In agreement with this idea, we have recently described in two distinct studies from Brazil that DM and also preDM were associated with increased frequency of clinical symptoms associated with TB [19]. Nevertheless, the association between $\mathrm{DM}$ and worse $\mathrm{TB}$ clinical and/or radiographic presentation has not been found by other investigations, including a recent study which examined Peruvian individuals [33]. It is possible that genetic or social differences between countries may influence the perception of the symptoms. Moreover, differences in timing from disease onset to admission at a TB clinical center may diminish the differences in clinical symptoms between normoglycemic and dysglycemic patients. Further studies are warranted to directly test these hypotheses. Interestingly, we found that TBDM patients exhibited increased AFB grades in sputum smears, which has been described by independent investigations worldwide $[3,19]$. These findings reinforce the idea that DM may impact capability to restrain mycobacterial growth and thus may be associated with increased risk of transmission.

Anemia is a common clinical condition associated with TB [34]. It has been recently reported that chronic anemia is linked to a distinct systemic inflammatory profile that persists after 2 months of ATT in a Brazilian cohort [26]. In addition, hemoglobin levels have been described to affect detection of glycated hemoglobin, which brings potential challenges in DM diagnosis in patients with severe anemia [26]. Herein, all the TB-DM patients were anemic (median Hb level: $9.9 \mathrm{~g} / \mathrm{dL}$ IQR: 7.6-13.4 g/dL). However, anemic status apparently did
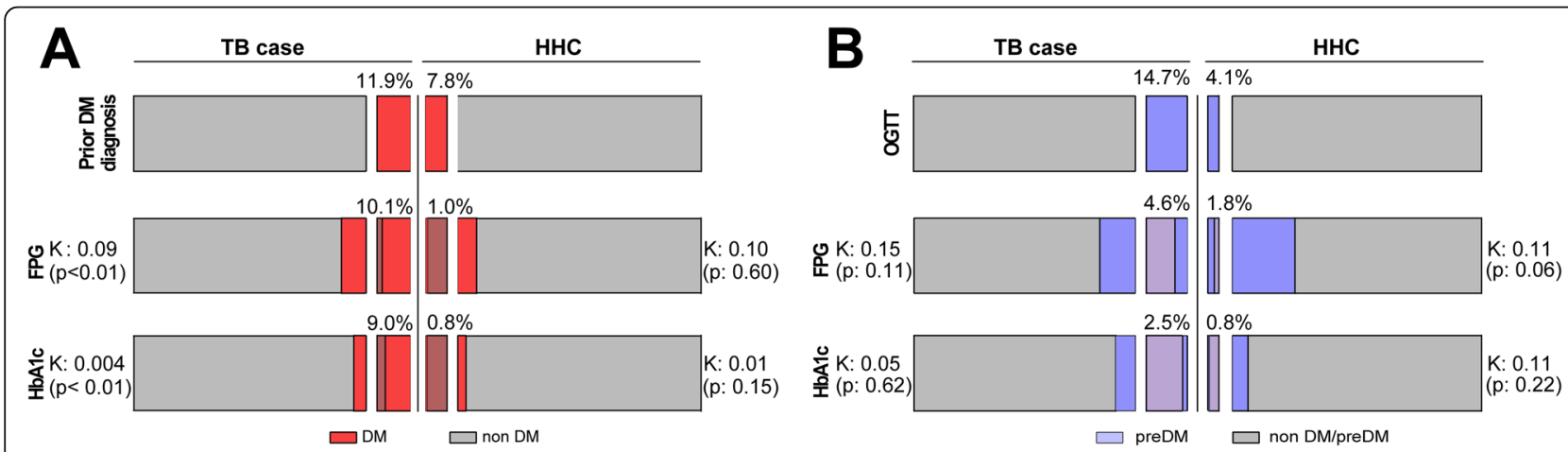

Fig. 6 Concordance Kappa (K) analysis between HbA1c, FPG and OGTT to detect diabetes or prediabetes in TB cases and HHC. a Results for TB cases (reference standard was prior DM diagnosis). b Results for HHC (reference value was OGGT). PD: Prior Diabetes Mellitus diagnosis; FPG: Fasting Plasma Glucose; HbA1c: Glycated Hemoglobin; OGTT: Oral Glucose Tolerance Test 
not prevent detection of $\mathrm{DM}$ in $\mathrm{TB}$ patients as the screening tests exhibited similar performance. Whether anemia impacts TB clinical presentation in DM patients is still unknown and deserves future investigation.

In our study, we found that all TB-DM people and a good proportion of TB-preDM patients were anemic. There is evidence supporting the idea that performance of HbA1c in detecting dysglycemia is affected by occurrence of anemia [35]. Herein, we analyzed the relationship between $\mathrm{HbA1c}$ and anemia and found no clear influence in the test performance to diagnose DM. Furthermore, our results demonstrated a low accuracy of the HbA1c vs. other tests to diagnose preDM, irrespective of the TB status. FPG was a better predictor of preDM than HbA1c in every condition tested. These observations suggest that FPG is indeed a good marker to be used for screening of DM or preDM in Peru, in addition to OGTT.

Our study has some limitations. OGTT was not tested on individuals who were recruited and a prior diagnosis of DM. This led to incomplete testing in all the study participants. In addition, we had study dropouts, which although were not very high, resulted in reduced number of individuals who were investigated in the latter study visits. Finally, the HbA1c levels could have been affected by the degree of anemia, as demonstrated before $[36,37]$. Our study was not designed to directly test the effect of anemia on changes in HbA1c levels and thus further research is warranted to address this question. Regardless of such limitations, our study results clearly show the high prevalence and heterogeneity of DM and preDM in a Peruvian population, corroborating with observations reported from other countries such as India [38].

\section{Conclusions}

The results presented here reveal a previously under notified high prevalence of DM and preDM in TB patients from Peru. Thus, screening strategies for both DM and preDM should be implemented in under routine program conditions for all patients. Using only HbA1c can lead to an error of sub-notification of preDM so the additional implementation of FPG and OGTT is needed in all cases where dysglycemia is suspected but not confirmed. Implementation of such screening tests in routine investigation at TB clinics could result in early detection of hyperglycemia and timely therapeutic interventions, which lead to improvements of clinical outcomes.

\section{Additional file}

Additional file 1: Table S1. Sensitivity and specificity analysis of the FPG and $\mathrm{HbA} 1 \mathrm{C}$ tests for detecting diabetes or prediabetes in the study population. (DOCX $19 \mathrm{~kb}$ )

\section{Abbreviations}

ADA: American Diabetes Association; AFB: Acid-Fast Bacilli;

ATT: Antitubercular treatment; BCG: Bacillus Calmette-Guérin; BMI: Body Mass Index; DM: Diabetes mellitus; FPG: Fasting plasma glucose; Hb: Hemoglobin; HbA1c: Glycated Hemoglobin; HHC: Household contact; IQR: Interquartile range; L-J: Löwenstein-Jensen; MDR: Multi Drug Resistant;

Mtb: Mycobacterium tuberculosis; NTP: National TB Program; OGTT: Oral glucose tolerance test; preDM: Prediabetes; ROC: Receiver Operator Characteristic; SEIS: Socios En Salud Informatic System; TB: Tuberculosis; WHO: World Health Organization

\section{Acknowledgments}

We thank the health workers in each of the participating health centers in Lima. We especially thank the patients and families that made this study possible.

\section{Authors' contributions}

RIC and MBA conceived and designed the study, interpreted the data, and wrote the manuscript. RIC, MBA, KL, NNB, OMS and LL implemented the lab study and collected the data. RIC, MBA, JFFN, DNA and BBA performed the data curation, analysis and performed data interpretation. All authors revised the manuscript critically for important intellectual content and gave final approval of the version to be published.

\section{Funding}

This work was mainly supported by the Consejo Nacional de Ciencia, Tecnología e Innovación Tecnológica (CONCYTEC-Peru) / Fondo Nacional de Desarrollo Científico, Tecnológico y de Innovación Tecnológica (FONDECYT, Convenio 175-2015). The work from BBA was supported by intramural research program from FIOCRUZ, by the National Institutes of Health (U01Al115940 and U01Al069923) and by the Departamento de Ciência e Tecnologia (DECIT) - Secretaria de Ciência e Tecnologia (SCTIE) - Ministério da Saúde (MS), Brazil (25029.000507/2013-07). MBA receives a fellowship from the Fundação de Amparo à Pesquisa da Bahia (FAPESB). BBA is a senior scientist from the Conselho Nacional de Desenvolvimento Científico e Tecnológico. The funders had no role in the design of the study and collection, analysis, and interpretation of data and in writing the manuscript.

\section{Availability of data and materials}

The datasets used and/or analysed during the current study available from the corresponding author on reasonable request.

\section{Ethics approval and consent to participate}

The study was approved by the Institutional Committee of Ethics for Humans (CIEl, approval number: 158-22-16), an autonomous committee established by the Universidad Peruana Cayetano Heredia, with the authorization of National Institute of Health in Peru. Written informed consent was obtained from all participants or their legally responsible guardians, and all clinical investigations were conducted according to the principles expressed in the Declaration of Helsinki and local and national Peruvian regulations.

\section{Consent for publication}

Not Applicable

\section{Competing interests}

The authors declare that they have no competing interest.

\section{Author details}

${ }^{1}$ Socios En Salud Sucursal Peru, 15001 Lima, Peru. ${ }^{2}$ Faculdade de Medicina, Universidade Federal do Rio de Janeiro, Rio de Janeiro 21941-590, Brazil. ${ }^{3}$ Faculdade de Medicina, Universidade Federal da Bahia, Salvador, Bahia 40110-100, Brazil. ${ }^{4}$ Instituto Brasileiro para Investigação da Tuberculose, Fundação José Silveira, Salvador, Bahia 40210-320, Brazil. ${ }^{5}$ nstituto Gonçalo Moniz, Fundação Oswaldo Cruz, Salvador, Bahia 40269-710, Brazil. ${ }^{6}$ Multinational Organization Network Sponsoring Translational and Epidemiological Research (MONSTER) Initiative, Fundação José Silveira, Salvador, Bahia 40210-320, Brazil. ${ }^{7}$ Curso de Medicina, Faculdade de Tecnologia e Ciências, Salvador, Bahia 41741-590, Brazil. ${ }^{8}$ Department of Global Health and Social Medicine, Harvard Medical School, Boston, MA 02115, USA. ${ }^{9}$ Universidade Salvador (UNIFACS), Laureate University, Salvador, 
Bahia 41720-200, Brazil. ${ }^{10}$ Escola Bahiana de Medicina e Saúde Pública, Salvador, Bahia 40290-000, Brazil.

Received: 14 May 2019 Accepted: 27 August 2019

Published online: 11 September 2019

\section{References}

1. GBD Tuberculosis Collaborators*. Global, regional, and national burden of tuberculosis, 1990-2016: results from the global burden of diseases, injuries, and risk factors 2016 study. Lancet Infect Dis. 2018;18(1):1329-49.

2. Jeon CY, Murray MB. Diabetes mellitus increases the risk of active tuberculosis: a systematic review of 13 observational studies. PLoS Med. 2008:5(7): 1152

3. Almeida-Junior JL, Gil-Santana L, Oliveira CA, Castro S, Cafezeiro AS, Daltro $C$, et al. Glucose metabolism disorder is associated with pulmonary tuberculosis in individuals with respiratory symptoms from Brazil. PLoS One. 2016:11(4):e0153590.

4. Shivakumar S, Chandrasekaran P, Kumar AMV, Paradkar M, Dhanasekaran K, Suryavarshini $\mathrm{N}$, et al. Diabetes and pre-diabetes among household contacts of tuberculosis patients in India: is it time to screen them all? Int I Tuberc Lung Dis. 2018;22(6):686-94.

5. Norma técnica de salud para la atención integral de las personas afectadas por tuberculosis, NTS N¹04-MINSA [ftp://ftp2.minsa.gob.pe/normaslegales/2 013/RM715_2013_MINSA.pdf]. Accessed 15 May 2019.

6. Chehregosha H, Khamseh ME, Malek M, Hosseinpanah F, Ismail-Beigi F. A view beyond $\mathrm{HbA1c}$ : role of continuous glucose monitoring. Diabetes Ther. 2019;10(3):853-63.

7. Hu Y, Liu W, Chen Y, Zhang M, Wang L, Zhou H, et al. Combined use of fasting plasma glucose and glycated hemoglobin A1c in the screening of diabetes and impaired glucose tolerance. Acta Diabetol. 2010;47(3):231-6.

8. Kumpatla S, Aravindalochanan V, Rajan R, Viswanathan V, Kapur A. Evaluation of performance of A1c and FPG tests for screening newly diagnosed diabetes defined by an OGTT among tuberculosis patients-a study from India. Diabetes Res Clin Pract. 2013;102(1):60-4.

9. Nakanga W, Crampin A, Nyirenda M. Personal view: should haemoglobin A1C be used for diagnosis of diabetes mellitus in Malawi? Malawi Med J. 2016:28(1):28-30.

10. Kumar PR, Bhansali A, Ravikiran M, Bhansali S, Dutta P, Thakur JS, et al. Utility of glycated hemoglobin in diagnosing type 2 diabetes mellitus: a community-based study. J Clin Endocrinol Metab. 2010;95(6):2832-5.

11. Miranda JJ, Bernabe-Ortiz A, Stanojevic S, Malaga G, Gilman RH, Smeeth L. $A 1 C$ as a diagnostic criteria for diabetes in low- and middle-income settings: evidence from Peru. PLoS One. 2011;6(3):e18069.

12. Ugarte-Gil C, Moore D. Comorbilidad de tuberculosis y diabetes: Problema aún sin resolver. Rev Peru Med Exp Salud Publica. 2014;31(1):137-42.

13. Manual para el diagnóstico bacteriológico de la Tuberculosis. Parte Baciloscopia [http://iris.paho.org/xmlui/bitstream/handle/123456789/782/ 9789275330135. pdf? sequence=1\&isAllowed=y]. Accessed 15 May 2019

14. Manual para el diagnóstico bacteriológico de la Tuberculosis. Parte 2 Cultivo [http://iris.paho.org/xmlui/bitstream/handle/123456789/18616/ tblabscultivo_2008.pdf?sequence=1\&isAllowed=y ]. Accessed 15 May 2019 .

15. Siddiqi S, Rüsch-Gerdes S. For BACTEC $C^{\mathrm{TM}}$ MGIT $960^{\mathrm{TM}}$ TB system; 2006.

16. International Diabetes Federation, IDF Diabetes Atlas [https://www.idf.org/ component/attachments/attachments.html?id=1093\&task=download]. Accessed 15 May 2019

17. Kliiman $\mathrm{K}$, Altraja A. Predictors of poor treatment outcome in multi- and extensively drug-resistant pulmonary TB. Eur Respir J. 2009;33(5):1085-94.

18. Brennan-Jones CG, Eikelboom RH, Bennett RJ, Tao KF, Swanepoel W. Asynchronous interpretation of manual and automated audiometry: agreement and reliability. J Telemed Telecare. 2018;24(1):37-43.

19. Gil-Santana L, Almeida-Junior JL, Oliveira CA, Hickson LS, Daltro C Castro $S$, et al. Diabetes is associated with worse clinical presentation in tuberculosis patients from Brazil: a retrospective cohort study. PLoS One. 2016:11(1):e0146876.

20. Prada-Medina CA, Fukutani KF, Pavan Kumar N, Gil -Santana L, Babu S, Lichtenstein F, et al. Systems immunology of DiabetesTuberculosis comorbidity reveals signatures of disease complications. Sci Rep. 2017;7(1):1999.

21. Situación de Tuberculosis en el Perú [www.tuberculosis.minsa.gob.pe/ portaldpctb/recursos/20180605122521.pdf]. Accessed 15 May 2019.

22. Ugarte-Gil C, Alisjahbana B, Ronacher K, Riza AL, Koesoemadinata RC, Malherbe ST, Cioboata R, Llontop JC, Kleynhans L, Lopez S, et al. Diabetes mellitus among pulmonary tuberculosis patients from four TB-endemic countries: the TANDEM study. Clin Infect Dis. 2019;ll:ciz28.

23. Popkin BM, Adair LS, Ng SW. Global nutrition transition and the pandemic of obesity in developing countries. Nutr Rev. 2012;70(1):3-21.

24. Diez-Canseco F, Saavedra-Garcia L. Programas Sociales y Reduccion de la Obesidad en el Perú: Reflexiones desde la investigación. Rev Peru Med Exp Salud Publica. 2018;34(1):105

25. Baker MA, Harries AD, Jeon CY, Hart JE, Kapur A, Lönnroth $\mathrm{K}$, et al. The impact of diabetes on tuberculosis treatment outcomes a systematic review. BMC Med. 2011;9:81.

26. Gil-Santana L, Cruz LAB, Arriaga MB, Miranda PFC, Fukutani KF, SilveiraMattos PS, et al. Tuberculosis-associated anemia is linked to a distinct inflammatory profile that persists after initiation of antitubercular therapy. Sci Rep. 2019;9(1):1381.

27. Aftab H, Ambreen A, Jamil M, Garred P, Petersen JH, Nielsen SD, et al. Comparative study of $\mathrm{HbA} 1 \mathrm{C}$ and fasting plasma glucose vs the oral glucose tolerance test for diagnosis of diabetes in people with tuberculosis. Diabet Med. 2017;34(6):800-3.

28. Grint D, Alisjhabana B, Ugarte-Gil C, Riza AL, Walzl G, Pearson F, et al. Accuracy of diabetes screening methods used for people with tuberculosis, Indonesia, Peru, Romania, South Africa. Bull World Health Organ. 2018; 96(11):738-49.

29. Vasudevan ARGS, Srivastava R, Premawardhana LD. Low HbA1c levels in a poorly controlled diabetic. Postgrad Med J. 2003;79(79):418.

30. Romanelli RJ, Chung S, Pu J, Nimbal V, Zhao B, Palaniappan L. Comparative effectiveness of early versus delayed metformin in the treatment of type 2 diabetes. Diabetes Res Clin Pract. 2015;108(1):170-8.

31. Kumar NP, Fukutani KF, Shruthi BS, Alves T, Silveira-Mattos PS, Rocha MS, et al. Persistent inflammation during anti-tuberculosis treatment with diabetes comorbidity. Elife. 2019;8:e46477.

32. Chiang CY, Bai KJ, Lin HH, Chien ST, Lee JJ, Enarson DA, et al. The influence of diabetes, glycemic control, and diabetes-related comorbidities on pulmonary tuberculosis. PLoS One. 2015;10(3):e0121698.

33. Ruslami R, Aarnoutse RE, Alisjahbana B, van der Ven AJ, van Crevel R. Implications of the global increase of diabetes for tuberculosis control and patient care. Trop Med Int Health. 2010;15(11):1289-99.

34. Hella J, Cercamondi Cl, Mhimbira F, Sasamalo M, Stoffel N, Zwahlen M, et al. Anemia in tuberculosis cases and household controls from Tanzania: contribution of disease, coinfections, and the role of hepcidin. PLoS One. 2018;13(4):e0195985

35. Huangfu P, Laurence $\mathrm{W}$, Alisjahbana B, Ugarte-Gil C, Riza AL, Walzl G, et al. Point of care HbA1c level for diabetes mellitus management and its accuracy among tuberculosis patients: a study in four countries. Int I Tuberc Lung Dis. 2019;23(3):283-92.

36. Silva JF, Pimentel AL, Camargo JL. Effect of iron deficiency anaemia on $\mathrm{HbA1c}$ levels is dependent on the degree of anaemia. Clin Biochem. 2016; 49(1-2):117-20

37. Urrechaga E. Influence of iron deficiency on $\mathrm{Hb}$ A1c levels in type 2 diabetic patients. Diabetes Metab Syndr. 2018;12(6):1051-5.

38. Kornfeld H, West K, Kane K, Kumpatla S, Zacharias RR, Martinez-Balzano C, et al. High prevalence and heterogeneity of diabetes in patients with TB in South India: a report from the effects of diabetes on tuberculosis severity (EDOTS) study. Chest. 2016;149(6):1501-8.

\section{Publisher's Note}

Springer Nature remains neutral with regard to jurisdictional claims in published maps and institutional affiliations.

\section{Ready to submit your research? Choose BMC and benefit from:}

- fast, convenient online submission

- thorough peer review by experienced researchers in your field

- rapid publication on acceptance

- support for research data, including large and complex data types

- gold Open Access which fosters wider collaboration and increased citations

- maximum visibility for your research: over $100 \mathrm{M}$ website views per year

At $\mathrm{BMC}$, research is always in progress.

Learn more biomedcentral.com/submission 